\title{
LOCALIZED MECHANICS OF DENTIN SELF-ETCHING ADHESIVE SYSTEM
}

\author{
Rodolfo Bruniera ANCHIETA ${ }^{1}$, Eduardo Passos ROCHA ${ }^{2}$, Ching-Chang KO ${ }^{3}$, Renato Herman SUNDFELD ${ }^{4}$, \\ Manoel MARTIN JUNIOR ${ }^{5}$, Carlos Marcelo ARCHANGELO ${ }^{5}$
}

\begin{abstract}
1- Undergraduate, Dental School of Araçatuba, São Paulo State University, Araçatuba, SP, Brazil.
2- Ph.D, Department of Dental Materials and Prosthodontics, Dental School of Araçatuba, São Paulo State University, Araçatuba, SP, Brazil. 3- Ph.D, Department of Orthodontics, The University of North Carolina at Chapel Hill, Chapel Hill, NC, USA.

4- Ph.D, Department of Restorative Dentistry, Dental School of Araçatuba, São Paulo State University, Araçatuba, SP, Brazil.

5- MS, Ph.D student, Department of Dental Materials and Prosthodontics, Dental School of Araçatuba, São Paulo State University, Araçatuba, SP, Brazil.
\end{abstract}

Corresponding address: Prof. Eduardo Passos Rocha - Departamento de Materiais Dentários e Prótese - Rua Jose Bonifacio, 1193 - 16015-050 Aracatuba, Sao Paulo, Brazil - Phone/Fax number: +55 18 3636-3245 - e-mail: eduardo rocha@foa.unesp.br

Received: June 12, 2007 - Accepted: August 02, 2007

\begin{abstract}
7

he bond strength of composite resins (CRs) to dentin is influenced by the interfacial microstructure of the hybrid layer (HL) and the resin tags (TAG). The contemporary self-etching primer adhesive systems overcame the inconvenient of the etch-andrinse protocol. Studies, however, have demonstrated that HL thickness and TAG length vary according to the wetting time and additional use of acid-etching prior to self-etching primers. This study investigated the localized stress distribution in the HL and the dentin/adhesive interface. Two HL thicknesses $(3$ or $6 \mu \mathrm{m})$, two TAG lengths $(13$ or $17 \mu \mathrm{m})$ and two loading conditions (perpendicular and oblique- $25^{\circ}$ ) were investigated by the finite element (FE) analysis. Five two-dimensional FE models (M) of a dentin specimen restored with CR $(38$ x $64 \mu \mathrm{m})$ were constructed: M1 - no HL and no TAG; M2 - $3 \mu \mathrm{m}$ of HL and $13 \mu \mathrm{m}$ of TAG; M3 $-3 \mu \mathrm{m}$ of HL and $17 \mu \mathrm{m}$ of TAG; M4 $-6 \mu \mathrm{m}$ of HL and $13 \mu \mathrm{m}$ of TAG; and M5 $-6 \mu \mathrm{m}$ of HL and $17 \mu \mathrm{m}$ of TAG. Two distributed loadings $(\mathrm{L})(20 \mathrm{~N})$ were applied on CR surface: L1 - perpendicular, and L2 - oblique $\left(25^{\circ}\right)$. Fixed interfacial conditions were assigned on the border of the dentin specimen. Ansys 10.0 (Ansys ${ }^{\circledR}$, Houston, PA, USA) software was used to calculate the stress fields. The peak of von Mises $\left(\sigma_{\mathrm{vM}}\right)$ and maximum principal stress $\left(\sigma_{\max }\right)$ was higher in L2 than in L1. Microstructures (HL and TAG) had no effect on local stresses for L1. Decreasing HL decreased $\sigma_{\mathrm{vM}}$ and $\sigma_{\max }$ in all structures for L2, but the TAG length had influence only on the peributular dentin. The thickness of HL had more influence on the $\sigma_{\mathrm{vM}}$ and $\sigma_{\max }$ than TAG length. The peritubular dentin and its adjacent structures showed the highest $\sigma_{\mathrm{vM}}$ and $\sigma_{\max }$, mainly in the oblique loading.
\end{abstract}

Uniterms: Dentin-bonding agents; Finite element analysis; Dentin; Dental acid etching.

\section{INTRODUCTION}

The bond of the composite resin to the dental substrate has been improved with the development of the adhesive resin systems. This should promote a better clinical performance of the restorative material, keeping the integrity and health of the tooth structures ${ }^{6}$

In the conventional techniques, the retention is obtained after etching the dentin with phosphoric acid for 20 seconds, in an etch-and-rinse protocol. This procedure removes the smear layer and smear plugs, exposing the dentinal tubules, and removes the mineral content of the superficial dentin, thereby exposing the type I collagen fibers from the dentin matrix. This condition creates a microporous surface to be penetrated by the adhesive resin that, by infiltrating the exposed collagen mesh, establishes the so-called hybrid layer $(\mathrm{HL})^{6}$.
Thus, the ideal HL is an integrated collagen/resin biopolymer that promotes a stable and continuous bond between the bulk adhesive layer and the intact dentin ${ }^{8}$. However, the adhesive potential of the dentin interface is still subject to failure due to the following reasons. Exposed collagen fibers require an adequate hydration level to prevent collapse. However, this hydration level is difficult to establish as too much drying could cause collapse. The process to control its hydrophilic nature, keeping the exposed collagen fibrils available to bond is not easy to establish. In addition, to reduce the failure at the dentin/ adhesive interface ( $d / a$ interface), the adhesive resin infiltration should be complete with no void incorporation, otherwise the stress level in the HL will be increased ${ }^{5}$.

Because of these limitations, the contemporary adhesives systems, such as the self-etching systems, were established to simplify the procedures and remove the 
multiple steps involved with the conventional protocol. These may be available as two-bottle system in which, the first bottle with a combination of the etchant and primer, and a separate bottle for the adhesive (self-etching primers), or as one-step system with a combination of the etchant, primer and adhesive resin in a single container (all-in-one adhesive systems).

The HL characteristics based on the contemporary selfetching systems may vary. Sundfeld, et al. ${ }^{9}$ (2005) showed different TAG lengths and HL thicknesses after changing the time of application (15, 30 or 45 seconds) of all-in-one adhesive systems. The changes in the TAG length were significant. In addition, the formed HL was significantly thicker (from 3.13 to $6.32 \mu \mathrm{m}$ ) when the self-etching adhesive system was applied to phosphoric acid-etched dentin. Although the additional etching on dentin has not been recommended to enhance the bond strength in dentin ${ }^{11}$, various HL thicknesses can also be obtained based only on the acidity of the self-etching system ${ }^{3}$.

It has been shown that the stress in a fully infiltrated HL is up to $10 \%$ smaller than that from a partial infiltration, including reduction in the stress concentration $z^{2} \mathrm{e}^{5}$. However, the stress levels based on different HL thicknesses and TAG lengths in a fully infiltration condition have not been properly reported. Based on the findings of Sundfeld, et al. ${ }^{9}$ (2005), this study investigated the hypothesis that the HL thickness has more influence in the stress distribution than TAG length. This work was designed to evaluate the stress distribution in the HL and $\mathrm{d} / \mathrm{a}$ interface by varying the HL thickness ( 3 and $6 \mu \mathrm{m}$ ), the TAG length (13 and $17 \mu \mathrm{m}$ ), and the loading condition (perpendicular and oblique), using two-dimensional finite element analysis.

\section{MATERIALAND METHODS}

Based on anatomic dimensions, five FE models were designed using varying the HL thicknesses and TAG lengths thus establishing models (M) 1 to 5. (Figures 1-3)

The material properties (Young's module $(E)$ and Poisson coefficient (n)) were established from literature (Figure 3). All materials were considered homogenous, isotropic and linearly elastic.

The adhesive resin was fully infiltrated through the collagen mesh. Misra, et al. ${ }^{5}$ (2004) showed that the intertubular dentin located near the HL has different mechanical properties from the intertubular dentin located far from the HL, a consequence of the gradual demineralization of the dentin. In order to reproduce this characteristic, a layer of intertubular dentin $(3 \mu \mathrm{m})$ was designed below the HL in groups 2 to 5 with specific mechanical properties (Figure 3 ).

The HL was idealized with the collagen being fully incorporated by the adhesive resin (Figure 1).

Misra, et al. ${ }^{5}$ (2004) showed that the exposed acid-etched collagen that is not infiltrated by the adhesive resin have a reduced $E(<2 \mathrm{GPa})$. In the present study, an $E$ of $2.5 \mathrm{GPa}$ was considered for the collagen fibers. First, because this is the average value from the interval used ( 1 to $4 \mathrm{GPa})^{5}$ and second, because this is higher than that established for non-infiltrated collagen.

The models were then entered to Ansys 10.0 software (Ansys Inc., Houston, PA, USA) for reconstruction of FE models. Plain-strain element (plane 2) was used in this study because it is a 6-node triangular element with a quadratic displacement behavior and is well suited to model irregular
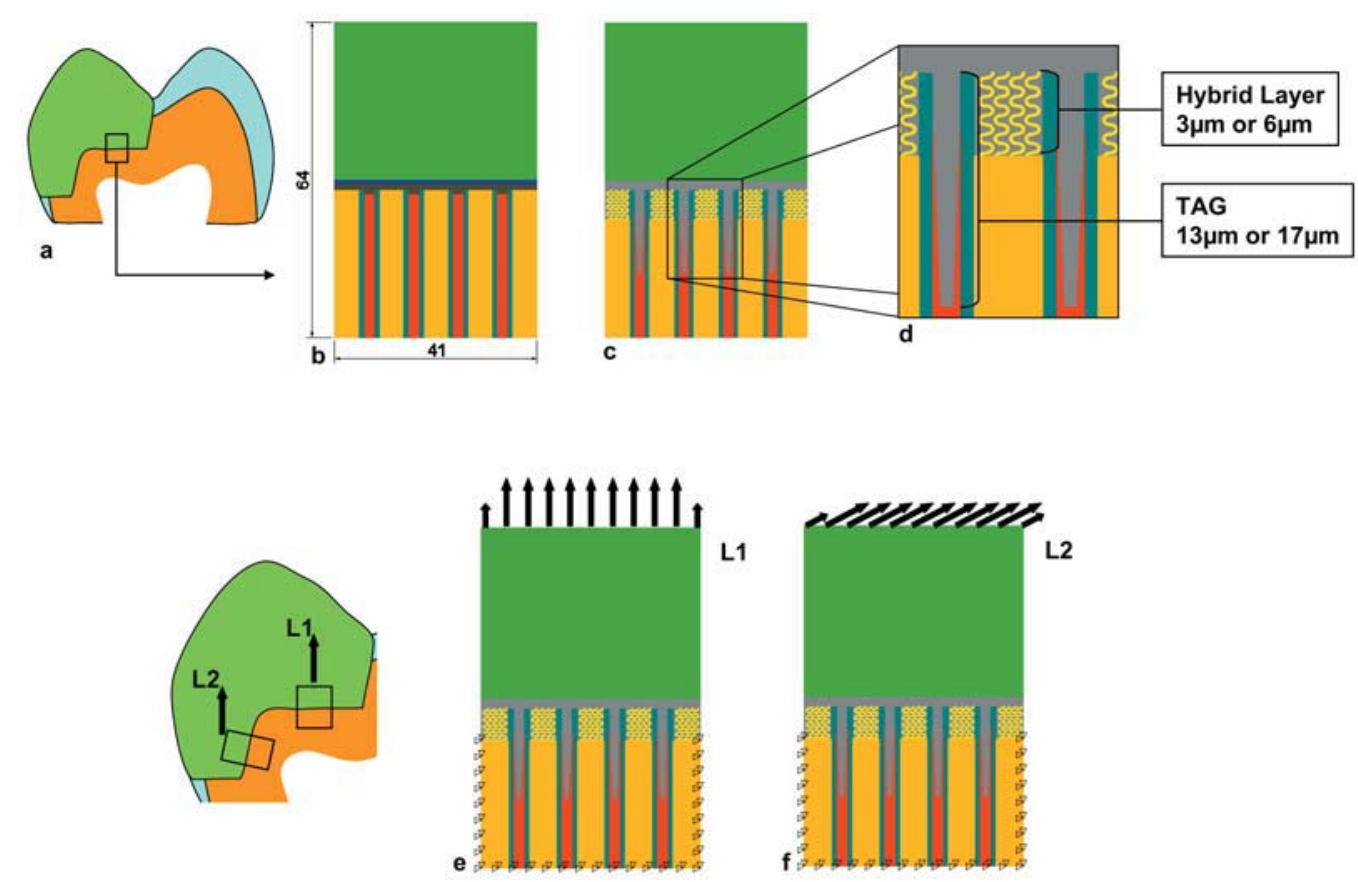

FIGURE 1- (a and b) Dentin specimen (orange) restored with composite resin (green) (dimensions in $\mu \mathrm{m}$ ). (c) Specimen with hybrid layer $(\mathrm{HL})$. (d) Variation of $\mathrm{HL}$ thickness (3 to $6 \mu \mathrm{m}$ ) and resin TAG length (13 to $17 \mu \mathrm{m})-\mathrm{collagen}$ (yellow), adhesive dentin and resin TAG (grey), peritubular dentin (dark green) and intertubular dentin (orange) (e) Perpendicular loading (L1). (f) Oblique loading (L2) 
meshes. The mesh was refined for the convergence of the solution.

For the boundary condition, all nodes on the bottom and lateral side of each model were fixed in $x$ and $y$ directions.

Distributed loading (L) distribution was performed in all nodes on the upper surface of the composite resin, in two conditions (Figure 1):

- L1 - Perpendicular load (traction) $(20 \mathrm{~N})$

- L2 - Oblique load $(20 \mathrm{~N})$ in $25^{\circ}$ with the composite resin surface.

von Mises $\left(\sigma_{\mathrm{vM}}\right)$ and maximum principal stress $\left(\sigma_{\max }\right)$ were used to predict failure of the structures. The peak value of $\sigma_{\mathrm{vM}}$ was calculated in each group and in the specific locations of interest, such as the intertubular dentin, peritubular dentin, adhesive layer and collagen fibers. The peak value of $\sigma_{\max }$ was calculated in the hybrid layer (M2 to M5).

\section{RESULTS}

The $\sigma_{\mathrm{vM}}$ stress in L1 was uniformly distributed throughout the structures without differences among groups. In view of this, the Figure 2 is based only on M2.

In L2, the stress was laterally concentrated. The peak of $\sigma_{\mathrm{vM}}$ was higher for L2 than that for L1 and it was located on the lateral surface of the peritubular dentin in contact with the TAG in all groups. (Figure 4)

The HL thickness and TAG length revealed no substantial influence in L1 for all groups. The effects of HL thickness and TAG length were observed only in L2. (Figure 5)

\section{Intertubular Dentin and Peritubular Dentin}

The peak of $\sigma_{\mathrm{vM}}$ in L2 was 6 times higher than that in L1. The lowest $\sigma_{\mathrm{vM}}$ value was observed in the thinner HL with shorter TAG (M2). However, the influence of the TAG length was only observed in thinner HL. (Figure 5)

The thicker HL promoted high $\sigma_{\mathrm{vM}}$ and the peak of $\sigma_{\mathrm{vM}}$ was located in the peritubular dentin (Figure 4). For intertubular dentin, it was located close to peritubular dentin.

\section{Adhesive Layer}

The peak of $\sigma_{\mathrm{vM}}$ in L2 was at least 4.5 times higher than that for L1. For the same thickness of HL, the TAG length had no influence on the $\sigma_{\mathrm{vM}}$ values (Figure 5). The $\sigma_{\mathrm{vM}}$ was higher for the thicker HL and it was located in the region close the top of the peritubular dentin (Figure 4). In addition, the $\sigma_{\mathrm{vM}}$ into resin TAG was more intense in L2. (Figure 5)

\begin{tabular}{|ccc|}
\hline MODELS & HL $(\boldsymbol{\mu m})$ & TAG $(\mu \mathrm{m})$ \\
\hline 1 & 0 & 0 \\
2 & 3 & $13 \mu \mathrm{m}$ \\
3 & 3 & $17 \mu \mathrm{m}$ \\
4 & 6 & $13 \mu \mathrm{m}$ \\
5 & 6 & $17 \mu \mathrm{m}$ \\
\hline
\end{tabular}

FIGURE 2- Models ( 1 to 5 ) with the HL and TAG dimensions (HL - Hybrid layer)

\begin{tabular}{|c|c|c|c|}
\hline STRUCTURES & DIMENSION ( $\mu \mathrm{m})$ & $E(\mathbf{G P a})^{5}$ & $\mathbf{n}^{4}$ \\
\hline \multirow{3}{*}{$\begin{array}{l}\text { Specimen Width } \\
\text { Length } \\
\text { Composite resin }\end{array}$} & 41 & & \\
\hline & 64 & & \\
\hline & 32 (length) & 30 & 0,3 \\
\hline Smear Layer ${ }^{6}$ & 1 (length) & $1^{*}$ & $0.3^{*}$ \\
\hline Smear Plug ${ }^{6}$ & 1 (length) & $1^{*}$ & $0.3^{*}$ \\
\hline Adhesive layer ${ }^{5}$ & 2 (length) & 5 & 0.28 \\
\hline \multirow[t]{2}{*}{$\mathrm{HL}$ - Collagen } & 3 (length) (M2 and M3) & 2.5 & 0.3 \\
\hline & 6 (length) (M4 and M5) & & \\
\hline \multirow[t]{2}{*}{$\mathrm{HL}$ - Adhesive layer ${ }^{9}$} & 3 (length) (M2 and M3) & 5 & 0.28 \\
\hline & 6 (length) (M4 and M5) & & \\
\hline Intertubular dentin close to $\mathrm{HL}$ & 3 (length) & 13 & 0.3 \\
\hline Intact intertubular dentin 5 & 5 (width) & 20 & 0.3 \\
\hline Peritubular dentin ${ }^{5}$ & 1 (width) & 28,6 & 0.3 \\
\hline Tubule $^{4}$ & 2 (width) & - & - \\
\hline \multirow[t]{2}{*}{ Resin TAG $^{9}$} & 13 (length) (M2 and M3) & 5 & 0.28 \\
\hline & 17 (length) (M4 and M5) & & \\
\hline
\end{tabular}

* An assumption to simulate low mechanical properties.

FIGURE 3- Dimensions (in $\mu \mathrm{m}$ ) and material properties ( $E$ and $\mathrm{n}$ ) 


\section{Collagen}

The peak of $\sigma_{\mathrm{vM}}$ in L2 was at least 4 times higher than that for L1. For the same HL thickness, the TAG length had no influence on the $\sigma_{\mathrm{vM}}$ values (Figure 5). The $\sigma_{\mathrm{vM}}$ was higher in the thicker HL. For M2 and M3 in L2, the peak of $\sigma_{\mathrm{vM}}$ was located on the upper half of the collagen fibers, and for M4 and M5 it was located on the lower half.

\section{$\sigma_{\max }$ in the Hybrid Layer}

The peak of $\sigma_{\max }$ was similar in L1 with no influence of HL thickness and TAG length. The $\sigma_{\max }$ values in L2 were 6 times higher than that for L1. (Figure 4)

The influence of HL thickness on the reduction of $\sigma_{\max }$ was more pronounced than the TAG length influence. (Figure 5)

\section{DISCUSSION}

It is well known that HL and TAG are responsible for the micromechanical retention in the adhesive restorations. An efficient HL should have the adhesive resin fully infiltrated through the collagen fibers exposed after the removal of mineral content of the dentin, with no void incorporation ${ }^{1}$.

This study idealized an adequate HL based on the selfetching system because the adhesive resin was fully infiltrated into the demineralized dentin, without voids. In addition to the intertubular dentin, the peritubular dentin, the bulk adhesive layer and the collagen fibers were perfectly bonded to each other. In this simplified HL, high stress was found in the peritubular dentin in all models. In sequence, the most requested structures were: the adhesive layer including adhesive TAG, the intertubular dentin and the collagen fibers.

In terms of $E$ influence, these results are in agreement with those of a previous report ${ }^{5}$, in which the structures with lowest $E$, such as the adhesive layer, transferred the stress to structures with higher $E$, such as the peritubular dentin in the present study.

In comparison to the thinner HL, it is expected that a thicker HL with longer TAG may provide some reduction of stress at the $d / a$ interface in a fully infiltrated condition. However, this hypothesis was not supported in this study. The stress in the peritubular and intertubular dentin was high in the thicker HL. Lower stress was observed in the thinner HL.

Furthermore, thinner HL and shorter TAG are obtained when the self-etching adhesive system is applied onto dentin with no modification in the application protocol ${ }^{9}$. Additionally, some studies have shown the occurrence of cohesive failures in dentin when the self-etching system is applied without prior acid etching. When the acid etching is performed, the adhesive failures at the $\mathrm{d} / \mathrm{a}$ interface are prevalent ${ }^{11}$.

In the present study, M2 had the characteristics (thinner HL and shorter TAG) based on the self-etching system without any modifications in the protocol. Moreover, the stress in M2 was usually lower than that in other models, mainly M4 and M5. (Figure 5) This means that no prior acid etching in dentin promotes not only better dentin bond strength $^{11}$, but also the reduction of the stress in the HL and $\mathrm{d} / \mathrm{a}$ interface. This reduction of stress might help keeping the integrity of the HL over time. This is largely due to the
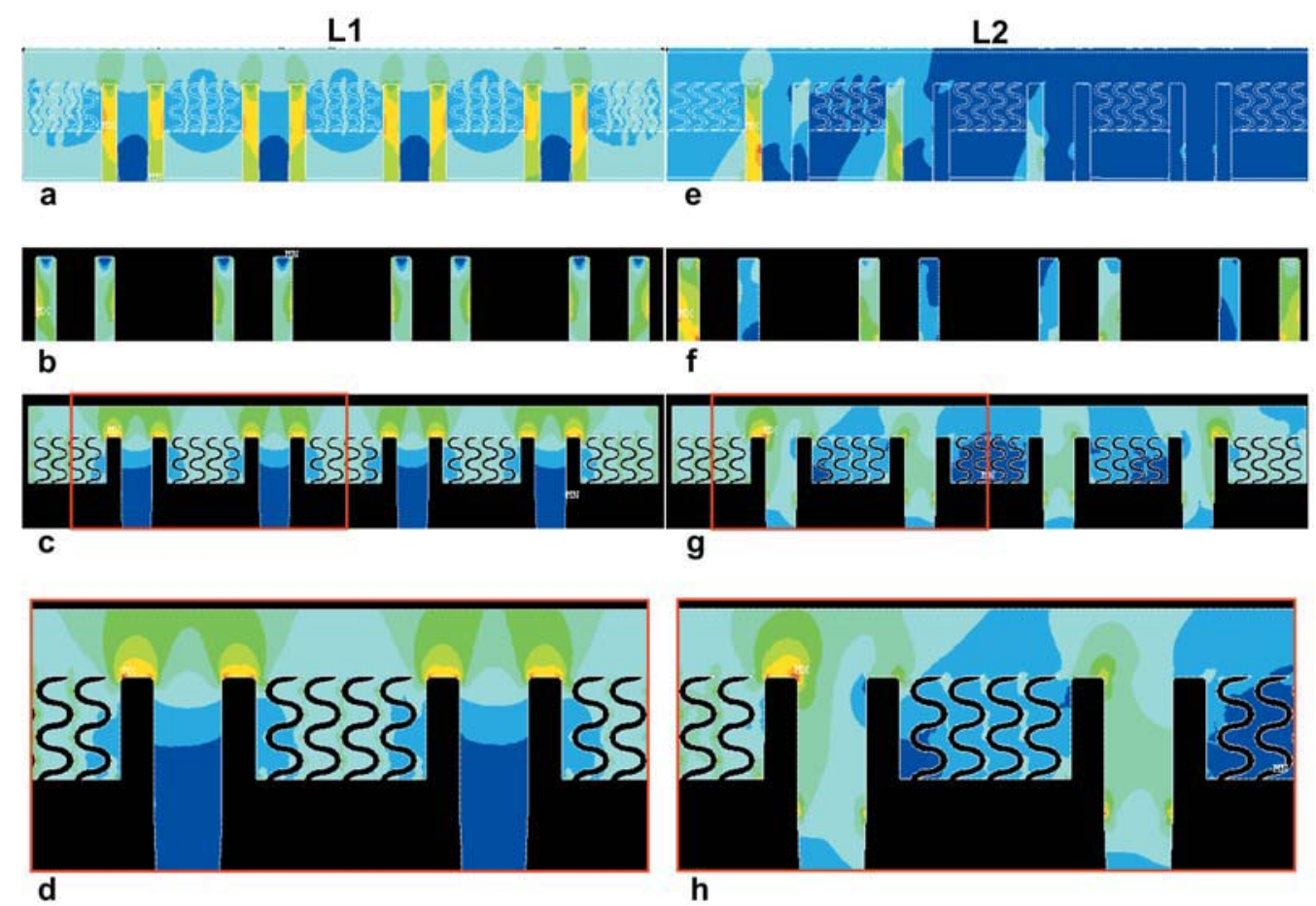

FIGURE 4- Stress distribution for $M 2$ regarding $L 1$ and L2. (a,e) $\sigma_{\max }(M P a)$ for $M 2$, (b,f) $\mathrm{S}_{\mathrm{vM}}(\mathrm{MPa})$ for peritubular dentin, (c,g) $\sigma_{\mathrm{vM}}$ $\left(\mathrm{MPa}\right.$ ) for adhesive layer (red box view in $\mathrm{d}$ and $\mathrm{h}$, respectively). Note the peak of $\sigma_{\mathrm{vM}}$ and $\sigma_{\text {max }}$ located laterally in the peritubular dentin (e and f) and more intense $\sigma_{\mathrm{vM}}$ in the adhesive layer and resin TAG (h) for L2 in comparison with L1 (d) 
benefits of the self-etching system. In this system, the adhesive exhibits a hydrophobic behavior that prevents hydrolysis because the bond is partially based on hydrophobic chemical interaction ${ }^{13}$.

With the previous acid etching of dentin, thicker HL and longer TAG are observed, as shown by Sundfeld, et al. ${ }^{9}$ (2005). In the present study, this condition showed high stress levels in all structures, mainly in the peritubular dentin. If the adhesive failure in dentin is prevalent when acid etching is performed ${ }^{11}$, the high stress level might severely reduce the bond quality over time. Thus, this can be one of the factors that explain why the bonding effectiveness of some self-etch adhesives systems to dentin is lower in comparison with the conventional adhesives systems ${ }^{10,12}$.

The thicker HL (M4 and M5) generated high stress in the peritubular dentin with no influence of TAG length. The TAG length had little effect when the HL was thin (M2 and M3). Similar results were observed in the intertubular dentin.

Regarding M1, in some moments, such as for peritubular dentin, the stress was higher than that observed in M2 M5 for L2. This was based on the linear aspect of this study which kept the smear layer perfectly bonded to dentin in M1. So, the lowest $E$ of smear layer allowed the transference of stress to the underneath structures with higher $E$, such as the peritubular dentin.

The influence of HL thickness on the adhesive layer and collagen fibrils were similar. The stress was lower in M2 and M3 in comparison to M4 and M5. Higher stress in the adhesive layer was related to thicker HL. In accordance with these results, the side effects, such as adhesive failure, might be higher if the thicker HL is established from prior acid etching protocol, which implies reduced dentin bond strength $^{11}$.

In general, the results showed higher stress in the peritubular dentin close to the HL and in the adhesive layer in contact with the top of the peritubular dentin. This is consistent with a previous study ${ }^{5}$ and shows that the thickness of HL is more important than the TAG length for stress reduction. However, a 3-D analysis should be performed to better understand this phenomenon, as the stress in those areas is likely to produce failure ${ }^{2,7}$ and might be higher than dentin bond strength values.

QL1 $\square$ L2
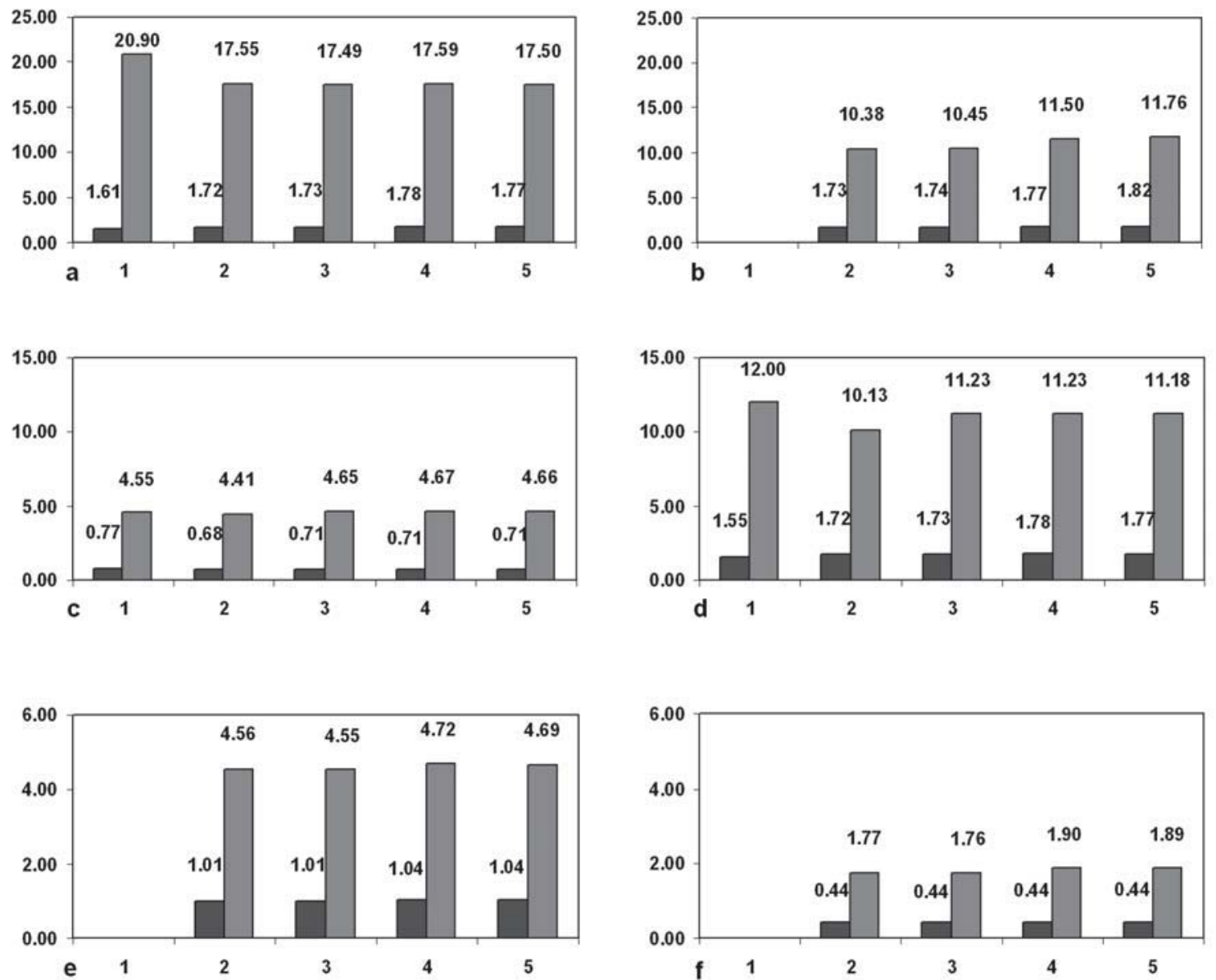

FIGURE 5- Stress values for each group (1 to 5) and each loading condition (L1 and L2). (a) Maximum $\sigma_{\mathrm{vM}}(\mathrm{MPa}),(\mathbf{b}) \sigma_{\max }(\mathrm{MPa})$ for hybrid layer, (c) $\sigma_{\mathrm{vM}}(\mathrm{MPa})$ for intertubular dentin, (d) $\sigma_{\mathrm{vM}}(\mathrm{MPa})$ for peritubular dentin, (e) $\sigma_{\mathrm{vM}}(\mathrm{MPa})$ for adhesive layer, and (f) $\sigma_{\mathrm{vM}}(\mathrm{MPa})$ for collagen 


\section{CONCLUSIONS}

The following conclusions may be drawn: 1 . The thicker HL was associated with high stress level. 2. The influence of TAG length was not revealed in the presence of thicker HL. In the presence of thinner HL, its influence was small. 3. The stress based on perpendicular loading was more uniform among structures than that obtained from oblique loading. 4. The peritubular dentin and its surroundings were the areas with highest stress concentration

\section{ACKNOWLEDGEMENT}

The authors would like to thank the FAPESP (São Paulo State Research Foundation - Protocol \#2006/01988-6) and CAPES (Protocol 2325-05-5) for the grants.

\section{REFERENCES}

1- Burrow MF, Takakura H, Nakajima M, Inai N, Tagami J, Takatsu T. The influence of age and depth of dentin on bonding. Dent Mater. 1994;10(4):241-6.

2- Jacques P, Hebling J. Effect of dentin conditioners on the microtensile bond strength of a conventional and a self-etching primer adhesive system. Dent Mater. 2005;21(2):103-9.

3- Kenshima S, Francci C, Reis A, Loguercio AD, Leonardo ER Filho. Conditioning effect on dentin, resin tags and hybrid layer of different acidity self-etch adhesives systems applied to thick and thin smear layer. J Dent. 2006;34(10):775-83.

4- Lee SY, Chiang HC, Huang HM, Shih YH, Chen HC, Dong DR, et al. Thermo-debonding mechanisms in dentin bonding systems using finite element analysis. Biomaterials. 2001;22(2):113-23.

5- Misra A, Spencer P, Marangos O, Wang Y, Katz JL. Micromechanical analysis of dentin/adhesive interface by the finite element method. J Biomed Mater Res. 2004;70(1):56-65.

6- Nakabayashi N, Pashley DH. Hybridization of dental hard tissues. Tokyo: Quintessence;1998.

7- Sidhu SK, Omata Y, Tanaka T, Koshiro K, Spreafico D, Semeraro $\mathrm{S}$, et al. Bonding characteristics of newly developed all-in-one adhesives systems. J Biomed Mater Res B Appl Biomater. 2007;80(2):297-303.

8- Spencer P, Wang Y. Adhesive phase separation at the dentin interface under wet bonding conditions. J Biomed Mater Res. 2002;62(3):44756

9- Sundfeld RH, Valentino TA, de Alexandre RS, Briso ALF, Sundefeld MLMM. Hybrid layer thickness and resin tag length of a self-etching adhesive bonded to sound dentin. J Dent. 2005;33(8):675-81.

10- Susin AH, Vasconcelos WA, Saad JRC, Oliveira OB Junior. Tensile bond strength of self-etching versus total-etching adhesive systems under different dentinal substrate conditions. Braz Oral Res. 2007;21(1):81-6.

11- Van Landuyt KL, Kanumilli P, De Munck J, Peumans M, Lambrechts P, Van Meerbeek B. Bond strength of a mild self-etch adhesive with and without prior acid-etching. J Dent. 2006;34(1):7785 .
12- Yesilyurt C, Bulucu B. Bond strength of total-etch and self-etch dentin adhesive systems on peripheral and central dentinal tissue: a microtensile bond strength test. J Contemp Dent Pract. 2006;7(2):2636

13- Yoshida Y, Nagakane K, Fukuda R, Nakayama Y, Okazaki M, Shintani H, et al. Comparative study on adhesive performance of functional monomers. J Dent Res. 2004;83(6):454-8. 